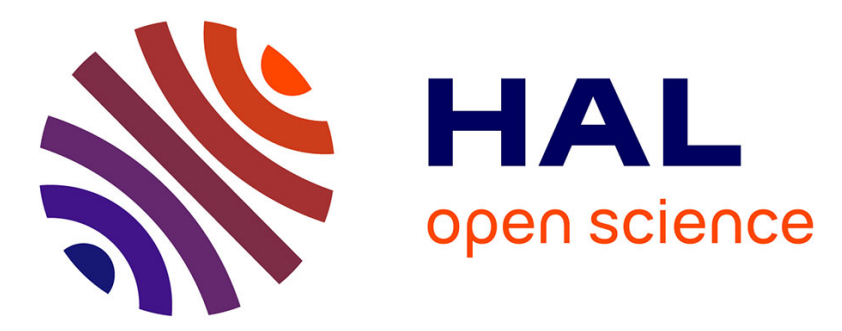

\title{
A priori error indicator in the transformation method for problems with geometric uncertainties
}

Duc Hung Mac, Stéphane Clenet, Jean-Claude Mipo, Igor Tsukerman

\section{To cite this version:}

Duc Hung Mac, Stéphane Clenet, Jean-Claude Mipo, Igor Tsukerman. A priori error indicator in the transformation method for problems with geometric uncertainties. IEEE Transactions on Magnetics, 2013, 49 (5), pp.1597 - 1600. 10.1109/TMAG.2013.2243706 . hal-00830870

\section{HAL Id: hal-00830870 https://hal.science/hal-00830870}

Submitted on 6 Jun 2013

HAL is a multi-disciplinary open access archive for the deposit and dissemination of scientific research documents, whether they are published or not. The documents may come from teaching and research institutions in France or abroad, or from public or private research centers.
L'archive ouverte pluridisciplinaire HAL, est destinée au dépôt et à la diffusion de documents scientifiques de niveau recherche, publiés ou non, émanant des établissements d'enseignement et de recherche français ou étrangers, des laboratoires publics ou privés. 


\title{
A priori error indicator in the transformation method for problems with geometric uncertainties
}

\author{
D. H. Mac ${ }^{1,2}$, S. Clénet ${ }^{1}$, J. C. Mipo², Igor Tsukerman ${ }^{3}$ \\ ${ }^{1}$ L2EP, Arts et Métiers ParisTech, 8 bd de Louis XIV, 59000 Lille, France \\ ${ }^{2}$ VALEO-Systèmes Electriques, Créteil, France \\ ${ }^{3}$ Department of Electrical and Computer Engineering, The University of Akron, OH 44325-3904, USA
}

\begin{abstract}
To solve stochastic problems with geometric uncertainties, one can transform the original problem in a domain with stochastic boundaries and interfaces to a problem defined in a deterministic domain with uncertainties in the material behavior. The latter problem is then discretized. There exist infinitely many random mappings that lead to identical results in the continuous domain but not in the discretized domain. In this paper, an $a$ priori error indicator is proposed for electromagnetic problems with scalar and vector potential formulations. This leads to criteria for selecting random mappings that reduce the numerical error. In an illustrative numerical example, the proposed $a$ priori error indicator is compared with an a posteriori estimator for both potential formulations
\end{abstract}

Index Terms-Stochastic problem, random domain, transformation method, random mapping, a priori error indicator, static fields.

\section{INTRODUCTION}

The Finite Element Method (FEM) is very widely used to model electro- and magnetostatic, quasistatic and electrodynamic behavior of electromagnetic devices. The required input data are the behavior laws of the material, the geometry of the device and the source terms.

In practice, the input data is available with some uncertainties due to the imperfections of the manufacturing processes, to the ageing of materials, to the environmental impact, etc. To take into account these uncertainties in accurate modeling of devices, a probabilistic approach can be used [1]. It consists in representing the uncertain input data by random variables. The output data of the model are then also random variables that need to be characterized.

In problems with geometric uncertainties, various probabilistic methods are available in the literature: the extended stochastic finite element method (XSFEM) [2] and the transformation method [3], [4] among others. The main idea of the transformation method consists in using a random mapping that transforms the initial problem into a reference problem defined on a deterministic domain. In the reference problem, the uncertainties are borne by the material behavior laws that become stochastic. Numerical tools are available to solve this kind of problem [5], [6].

In the transformation method, there exist an infinite number of random mappings that lead to the same solution in the continuous domain. In the reference domain discretized by FEM, the solutions depend on the mapping and are subject to numerical errors (the difference between the numerical solution and the exact solution). It is important to develop numerical measures and tools for selecting random mappings that lead to higher accuracy. This paper proposes such a tool: an a priori error indicator associated with stochastic mappings. In the mathematical development, the mapping is assumed to be piecewise-linear as an approximation, but the results can be extended to higher-order approximations.

In the first part of the paper, we introduce a magnetostatic problem with geometric uncertainties and also a transformation method. We then discuss an a priori error indicator in the transformation method with both scalar and vector potential formulations. Finally, the validity of the proposed a priori error indicator is tested in a magnetostatic example by comparison with an a posteriori error estimator.

\section{Magnetostatic Problem with Geometric Uncertainties - A Transformation Method}

\section{A. Magnetostatic problem with geometric uncertainties}

We are interested in a magnetostatic problem defined in a domain $D$.

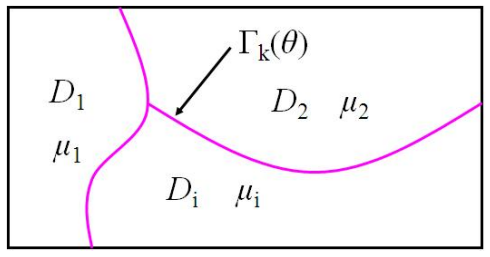

Figure 1: Magnetostatic problem defined in a domain $D$.

We assume that domain $D$ is split up into $n_{D}$ subdomains $D_{i}$, in each of which the permeability $\mu_{i}$ is assumed to be constant. The interfaces $\Gamma_{k}(\theta)$ between the subdomains are random, and parameter $\theta$ refers to a random outcome. We also assume that the random boundaries $\Gamma_{k}(\theta)$ can be expressed explicitly as functions of a vector $\xi(\theta)$ of independent random variables with known probability density functions (pdf's). For brevity, the dependence of the random vector $\xi$ on $\theta$ will not be explicitly indicated. The permeability $\mu(x, \xi)$ in the domain $D$ can be written in the following form:

$$
\mu(x, \xi)=\sum_{i=1}^{n_{D}} \mu_{i} I_{D_{i}}(x, \xi)
$$

where $I_{D_{i}}(x, \xi)$ is the indicator function equal to one within $D_{i}$ and zero otherwise. The magnetostatic equations defined 
on $D$, in the absence of sources, can be written as

$$
\left\{\begin{array}{c}
\nabla \cdot \mathbf{B}(x, \xi)=0 \\
\nabla \times \mathbf{H}(x, \xi)=0 \\
\mathbf{B}(x, \xi)=\mu(x, \xi) \mathbf{H}(x, \xi)
\end{array}\right.
$$

where $\mathbf{H}(x, \xi)$ and $\mathbf{B}(x, \xi)$ are the magnetic field and the magnetic flux density, respectively. Problem (2) is supplemented with boundary conditions. Generally, the scalar potential $\Omega(x, \xi)$ (such that $\mathbf{H}(x, \xi)=-\nabla \Omega(x, \xi)$ ) or the vector potential $\mathbf{A}(x, \xi)$ (such that $\mathbf{B}(x, \xi)=\nabla \times \mathbf{A}(x, \xi))$ are used to solve the problem. In the remainder, we briefly describe the transformation method for (2).

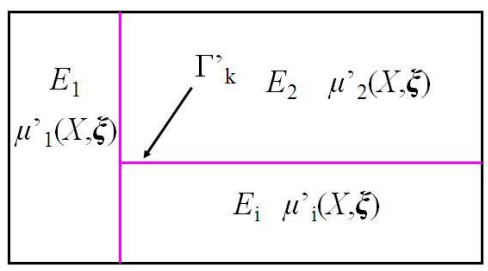

Figure 2: Magnetostatic problem defined in the reference domain $E$.

\section{B. Transformation method}

The main idea consists in transforming a problem with geometric uncertainties (random interfaces and boundaries in $D$ ) into a problem in a reference domain $E$ with deterministic interfaces and boundaries. In the transformed problem, the randomness is borne by the behavioral law. One needs to define a one-to-one random mapping $X(x, \xi)$ that transforms random interfaces $\Gamma_{k}(\xi)$ into deterministic interfaces $\Gamma_{k}^{\prime}$. It can be shown [4], [12] that the problem defined on $E$ (the reference problem) is equivalent to the problem defined on $D$ (the original problem) if the permeability $\mu^{\prime}(X, \xi)$ in $E$ has the following form:

$$
\mu^{\prime}(X, \xi)=\frac{M^{T}(X, \xi) \mu(X) M(X, \xi)}{|\operatorname{det} M(X, \xi)|}
$$

where $M(X, \xi)$ is the Jacobian matrix of the mapping $X(x, \xi)$. Note that capital $X$ denotes a point in the reference domain, while small $x$ is a point in the original domain.

In the case of scalar potentials,

$$
\Omega_{\mathrm{ex}}(x, \xi)=\Omega_{\mathrm{ex}}^{\prime}(X(x, \xi), \xi)
$$

where $\Omega_{\mathrm{ex}}$ and $\Omega_{\mathrm{ex}}^{\prime}$ are the exact solutions of the problems on $D$ (Fig. 1) and on $E$ (Fig. 2), respectively. Therefore, the solution of the original problem can be obtained from the solution of the reference problem via (4).

The main difficulty of the transformation method lies in the definition of the random mapping. In [4], a few relevant methods are discussed. Different mappings lead to different discrete problems on $E$ because different Jacobian matrices $M(X, \xi)$ lead to different permeabilities $\mu^{\prime}(X, \xi)$ (3). On the continuous level, the transformed problems are equivalent to the original ones, but this is no longer the case after discretization. The numerical solutions of the reference problems can be different and therefore the numerical errors depend on the mapping. In the remainder, an a priori error indicator in terms of the Jacobian matrix of the mapping is proposed and applied to evaluate different mappings.

\section{A priori ERROR INDICATOR}

The reference problem is solved numerically by introducing in the domain $E$ a tetrahedral mesh $\mathcal{M}$ with $n_{0}$ nodes, $n_{1}$ edges, $n_{2}$ facets and $n_{3}$ elements. We denote with $h$ the maximum radius of the circumscribed spheres of the elements. The piecewise-linear mapping (assumed as an approximation) has, for each $\xi$, a constant Jacobian matrix $M_{i}(\xi)$ over each tetrahedral element $e_{i}$ of $\mathcal{M}$. In this section, we aim at estimating the error for the scalar and vector potential formulations.

\section{A. Error estimation for the scalar potential formulation}

1) Definition of numerical error.

We introduce the following functional spaces:

$$
\begin{gathered}
H^{1}(E)=\left\{\left.u(X) \in L^{2}(E)\left|\int_{E}\right| \nabla u(X)\right|^{2} d X<\infty\right\} \\
V_{h}(E)=\operatorname{span}\left\{w_{0 i}(X) \mid i=1,2, \ldots, n_{0}\right\}
\end{gathered}
$$

$w_{0 i}$ being the first order interpolation function associated with node $i$. The exact solution $\Omega_{\mathrm{ex}}^{\prime}(X(x, \xi), \xi)$ of the scalar potential formulation satisfies

$$
\int_{E} \mu^{\prime}(X, \xi) \nabla \Omega_{\mathrm{ex}}^{\prime}(X, \xi) \cdot \nabla \lambda(X) d X=0,
$$

$\forall \lambda(X) \in H^{1}(E)$. We also define $\Omega_{h}^{\prime}(X, \xi)$ such that

$$
\int_{E} \mu^{\prime}(X, \xi) \nabla \Omega_{h}^{\prime}(X, \xi) \cdot \nabla w_{0 i}(X) d X=0,
$$

$\forall w_{0 i}(X) \in V_{h}(E)$. To have a well posed problem, a gauge condition should be added (the value of the scalar potential should be prescribed at a point). Note that the final numerical solution is obtained from $\Omega_{h}^{\prime}(X, \xi)$ after a discretization at the stochastic level using a polynomial chaos expansion [7]. In this paper, we are interested only in the error due to the spatial discretization. This error $\operatorname{er}(\xi)$ is defined as

$$
\begin{aligned}
\operatorname{er}^{2}(\xi)= & \int_{E} \mu^{\prime}(X, \xi) \nabla\left(\Omega_{h}^{\prime}(X, \xi)-\Omega_{\mathrm{ex}}^{\prime}(X, \xi)\right) \\
& \cdot \nabla\left(\Omega_{h}^{\prime}(X, \xi)-\Omega_{\mathrm{ex}}^{\prime}(X, \xi)\right) d X
\end{aligned}
$$

As already noted, for a domain $E$ and a given mesh $\mathcal{M}$, the permeability $\mu^{\prime}(X, \xi)(3)$ and then the potential $\Omega_{h}^{\prime}(X, \xi)$ depend on the choice of the mapping. Therefore the numerical error also depends on the mapping, as described in the following sections.

2) A priori error indicator.

Based on the development in [8], [9], we obtain the following error estimate:

$$
\operatorname{er}^{2}(\xi) \leq \sum_{i=1}^{n_{3}} \operatorname{er}_{i}^{2}(\xi)
$$

with

$$
\begin{gathered}
\operatorname{er}_{i}^{2}(\xi)=\frac{v_{e_{i}}}{\lambda_{\text {min }}^{i}(\xi) \sqrt{\lambda_{\text {min }}^{i}(\xi) \lambda_{\text {mid }}^{i}(\xi) \lambda_{\text {max }}^{i}(\xi)}} \\
\cdot\left[\sqrt{C_{1}(\xi)}+\sqrt{C_{2}(\xi) \lambda_{0 \max }^{i} \lambda_{\max }^{i}(\xi) / \lambda_{\min }^{i}(\xi)} h_{e_{i}}\right]^{2} h_{e_{i}}^{2}
\end{gathered}
$$


where $\lambda_{\max }^{i}(\xi) \geq \lambda_{\text {mid }}(\xi)^{i} \geq \lambda_{\min }^{i}(\xi)$ are the three eigenvalues of matrix $M_{i}^{T}(\xi) M_{i}(\xi), v_{e i}$ is the volume and $h_{e i}$ is the radius of the circumscribed sphere of element $e_{i}$. The coefficients $C_{1}(\xi)$ and $C_{2}(\xi)$ are independent of the random mapping and depend only on the exact solution of the original problem in $D$. The coefficient $\lambda_{0 \max }^{i}$ is the maximum eigenvalue of the $4 \times 4$ matrix $G^{i}$ whose coefficients are given by:

$$
G_{k l}^{i}=v_{e i}^{-1} \int_{e_{i}} \nabla w_{0 k}(X) \cdot \nabla w_{0 l}(X) d X
$$

It is shown in [8], [9] that the coefficient $\lambda_{0 \max }^{i}$ characterizes mesh quality. Moreover, the eigenvalues are related by the following conditions:

$$
\begin{aligned}
& v_{D}(\xi)=\sum_{i=1}^{n_{3}} \int_{e_{i}} \operatorname{det}^{-1} M_{i}(\xi) d X \\
& =\sum_{i=1}^{n_{3}} \frac{v_{e_{i}}}{\sqrt{\lambda_{\text {max }}^{i}(\xi) \lambda_{\text {mid }}^{i}(\xi) \lambda_{\text {min }}^{i}(\xi)}}
\end{aligned}
$$

Since the volume $v_{D}(\xi)$ is independent of the choice of the mapping, the sum in (12) is also independent of this choice. It is obvious that $v_{e_{i}}$ depends only on the mesh $\mathcal{M}$ and not on the mapping.

For a given reference domain $E$ with a given mesh, according to (10), one can expect that a mapping with the smallest ratios between $\lambda_{\max }^{i}$ and $\lambda_{\min }^{i}$ should tend to produce the smallest numerical error. We propose this as a practical criterion for comparing different mappings.

The error indicator above applies to piecewise-linear mappings. An arbitrary mapping can be approximated by a piecewise-linear one by standard first order finite-element-like interpolation of the nodal values. Moreover, the error indicator can be extended to higher-order approximations using e.g. the general criterion of [8].

\section{B. Error estimation for the vector potential formulation}

1) Definition of numerical error.

We introduce the functional space

$$
H_{\mathrm{curl}}(E)=\left\{\mathbf{H}(X) \in\left(L^{2}(E)\right)^{3}\left|\int_{D}\right| \nabla \times\left.\mathbf{H}(X)\right|^{2} d X<\infty\right\}
$$

With the mesh $\mathcal{M}$, we define the following discrete functional space:

$$
V_{1 h}(E)=\operatorname{span}\left\{\mathbf{w}_{1 i}(X) \mid i=1,2, \ldots, n_{1}\right\}
$$

$\mathbf{w}_{1 i}$ being the basis function associated with edge $i$ [13]. The exact vector potential $\mathbf{A}_{\mathrm{ex}}^{\prime}(X, \xi)$ is defined by

$$
\int_{E}\left(\left(\mu^{\prime}\right)^{-1}(X, \xi) \nabla \times \mathbf{A}_{\mathrm{ex}}^{\prime}(X, \xi)\right) \cdot \nabla \times \mathbf{w}(X) d X=0,
$$

$\forall \mathbf{w}(X) \in H_{\text {curl }}(E)$. We also define $\mathbf{A}_{h}^{\prime}(X, \xi)$ such that

$$
\int_{E}\left(\left(\mu^{\prime}\right)^{-1}(X, \xi) \nabla \times \mathbf{A}_{h}^{\prime}(X, \xi)\right) \cdot \nabla \times \mathbf{w}_{1 i}(X) d X=0,
$$

$\forall \mathbf{w}_{1 i}(X) \in V_{1 h}(E)$. The numerical error is defined by

$$
\operatorname{er}^{\prime 2}(\xi)=\int_{E}\left(\left(\mu^{\prime}\right)^{-1}(X, \xi) \nabla \times\left(\mathbf{A}_{h}^{\prime}(X, \xi)-\mathbf{A}_{\mathrm{ex}}^{\prime}(X, \xi)\right)\right)
$$

$$
\cdot \nabla \times\left(\mathbf{A}_{h}^{\prime}(X, \xi)-\mathbf{A}_{\mathrm{ex}}^{\prime}(X, \xi)\right) d X
$$

2) A priori error indicator.

With the same process as in Section III-A, we can obtain:

$$
\operatorname{er}^{\prime 2}(\xi) \leq \sum_{i=1}^{n_{3}} \operatorname{er}_{i}^{\prime 2}(\xi)
$$

with

$$
\begin{gathered}
\operatorname{er}_{i}^{\prime 2}(\xi)=\left[\sqrt{C_{1}^{\prime}(\xi)}+\sqrt{\lambda_{\text {max }}^{i}(\xi) \lambda_{\text {mid }}^{i}(\xi) / \lambda_{\text {min }}^{i}(\xi)} \sqrt{C_{2}^{\prime}(\xi) \lambda_{1 \max }^{i}} h_{e_{i}}^{2}\right]^{2} \\
\cdot \frac{v_{e_{i}} h_{e_{i}}^{2}}{\lambda_{\text {min }}^{i}(\xi) \sqrt{\lambda_{\text {min }}^{i}(\xi) \lambda_{\text {mid }}^{i}(\xi) \lambda_{\text {max }}^{i}(\xi)}}
\end{gathered}
$$

Minimizing the ratio between $\lambda_{\max }^{i}$ and $\lambda_{\min }^{i}$ will again tend to reduce the numerical error.

\section{Application}

We now illustrate the conclusion drawn in the previous section. We consider the magnetostatic problem defined in Fig. 3. The domain $D$ is split up into three sub-domains with the permeabilities $\mu_{1}=\mu_{2}=1000, \mu_{3}=1$. A magnetomotive force $\gamma_{0}$ is imposed between $\Gamma_{1}$ and $\Gamma_{2}$. Dimensions $c_{1,2}$ are assumed to be random and to belong to a given interval.

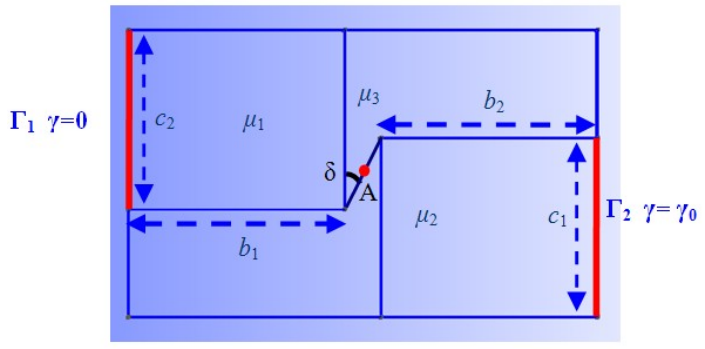

Figure 3: Original magnetostatic problem defined in domain $D$.

To characterize the deformation, we introduce the $\delta$ angle as defined in Fig. 3. We compare two mappings $T_{1}$ and $T_{2}$ presented in Figs. 4 and 5. We are interested in realizations of $\operatorname{cotan} \delta$ that depend directly on the values of $c_{1,2}$ (Fig. 4).

For each value of $\operatorname{cotan} \delta$, we solve numerically two reference problems corresponding to two mappings $T_{1}$ and $T_{2}$. Fig. 6 presents the evolution of the ratio $r=\lambda_{\max }^{A} / \lambda_{\min }^{A}$ between the maximum and minimum eigenvalues of $M^{T} M$ at point $\mathrm{A}$ as a function of $\operatorname{cotan} \delta$. This ratio is much greater for $T_{1}$ than for $T_{2}$. This is not surprising because mapping $T_{2}$ is simply a dilation - compression of each subdomain $D_{i}$ that yields a diagonal Jacobian matrix $M$. In contrast, mapping $T_{1}$ presents a shear distortion in sub-domains $D_{3}, D_{4}$ that results in a Jacobian matrix $M$ with significant off-diagonal terms and a large ratio $r$ between the maximum and minimum eigenvalues of $M^{T} M$. In Fig. 7, we plot an a posteriori error estimate that evaluates the sum of $\operatorname{er}(\xi)$ and $\operatorname{er}^{\prime}(\xi)$ as a function of $\delta$.

An a posteriori error $\mathrm{er}_{p}$ is obtained from the numerical solutions of the scalar and vector potential formulations:

$\mathrm{er}_{p}^{2}=\int_{E} \mu^{\prime}\left(\nabla \Omega_{h}^{\prime}-\left(\mu^{\prime}\right)^{-1} \nabla \times \mathbf{A}_{h}^{\prime}\right) \cdot\left(\nabla \Omega_{h}^{\prime}-\left(\mu^{\prime}\right)^{-1} \nabla \times \mathbf{A}_{h}^{\prime}\right) d X$ 


$$
\begin{gathered}
=\int_{E} \mu^{\prime} \nabla\left(\Omega_{h}^{\prime}-\Omega_{\mathrm{ex}}^{\prime}\right) \cdot \nabla\left(\Omega_{h}^{\prime}-\Omega_{\mathrm{ex}}^{\prime}\right) d X \\
+\int_{E}\left(\mu^{\prime}\right)^{-1} \nabla \times\left(\mathbf{A}_{h}^{\prime}-\mathbf{A}_{\mathrm{ex}}^{\prime}\right) \cdot \nabla \times\left(\mathbf{A}_{h}^{\prime}-\mathbf{A}_{\mathrm{ex}}^{\prime}\right) d X
\end{gathered}
$$

This estimator enables one to determine the sum of the numerical errors of the two complementary solutions [10], [11]. There is a very good correlation between the evolution of the error $\mathrm{er}_{p}$ in Fig. 7 and the ratio $r$ as a function of $\operatorname{cotan} \delta$ in Fig. 6, illustrating that the criterion $r$ can be used to choose the best mapping.

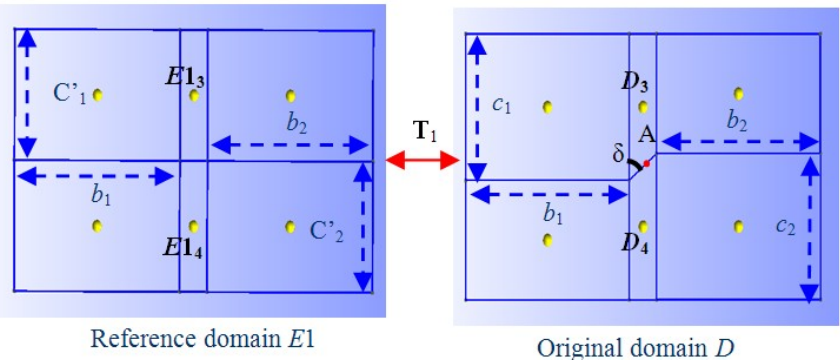

Figure 4: The reference domain $E 1$ associated with the mapping $T_{1}$ and the correspondence between the subdomains $E 1_{i}$ and $D_{i}$.



Reference domain $E 2$

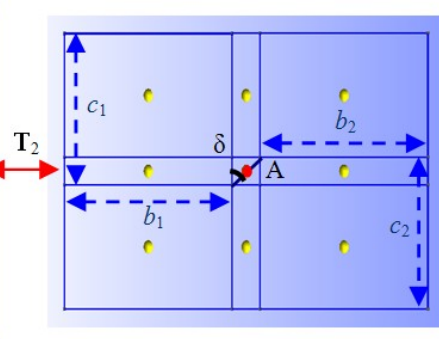

Original domain $D$
Figure 5: The reference domain $E 2$ associated with the mapping $T_{2}$ and the correspondence between the subdomains $E 2_{i}$ and $D_{i}$.

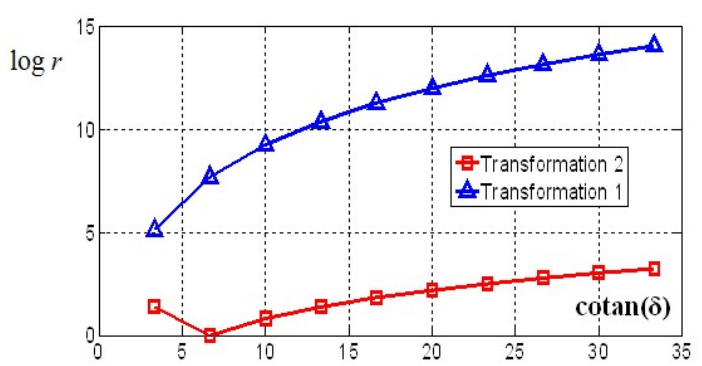

Figure 6: Evolution of the ratio $r$ as a function of $\operatorname{cotan} \delta$.

\section{Conclusion}

We have presented an a priori error indicator for the finite element - stochastic transformation method. The numerical error is estimated via the ratio $r$ of the max/min singular values of the Jacobian matrix $M$ of the transformation. The stochastic

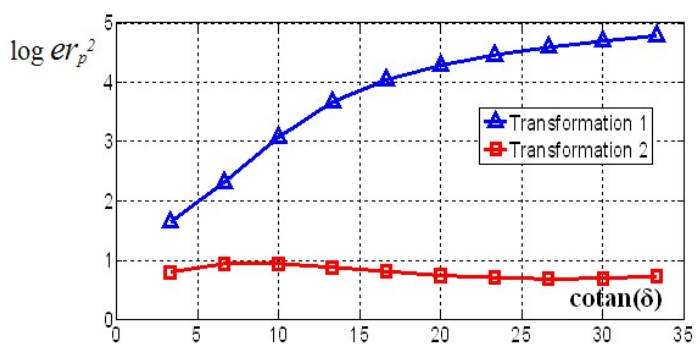

Figure 7: Evolution of the numerical errors as a function of $\operatorname{cotan} \delta$.

mapping is not unique but can be judiciously chosen using the new error indicator. From the numerical example given in the paper, one concludes that a mapping with dilation compression is preferable to a mapping with shear distortions.

\section{REFERENCES}

[1] R. Ghanem, P. Spanos. "Stochastic finite elements: a spectral approach," Springer-Verlag, Berlin, 1991.

[2] A. Nouy, A. Clement, F. Schoefs, and N. Moes. "An extended stochastic finite element method for solving stochastic differential equations on random domains," Computer Methods in Applied Mechanics and Engineering, Vol. 197, no. 51-52, pp. 4663-4682, 2008.

[3] D. Xiu, D.M. Tartakovsky. "Numerical methods for differential equations in random domains," SIAM J. Sci. Comput., Vol. 28, no. 3, pp. 1167 1185, 2006.

[4] D.H. Mac, S. Clenet, J.C. Mipo. "Transformation method for static field problem with random domain," IEEE Transactions on Magnetics, Vol. 47, no. 5, pp. 1446-1449, 2011.

[5] R. Gaignaire, S. Clenet, O. Moreau, and B. Sudret. "3D spectral stochastic finite element method in electromagnetism," IEEE Transactions on Magnetics, Vol. 43, no.4, pp. 1209-1212, 2007.

[6] K. Beddek, Y. Le Menach, S. Clenet, O. Moreau. "3D spectral finite element in static electromagnetism using vector potential formulation," IEEE Transactions on Magnetics, Vol. 47, no. 5, pp. 1250-1253, 2011.

[7] D. Xiu, G.E. Karniadakis. "The Wiener-Askey polynomial chaos for stochastic differential equations," SIAM J. Sci. Comput, Vol. 24, no. 2, pp. 619-644, 2002.

[8] Igor Tsukerman. "A general accuracy criterion for finite element approximation," IEEE Transactions on Magnetics, Vol. 34, no. 5, 1998.

[9] Igor Tsukerman. "Computational Methods for Nanoscale Apllications Particles, Plasmons and Waves," Nanostructure science and technology, Springer 2007

[10] J. Penman, J.R. Fraser. "Complementary and dual energy finite element principles in magnetostatics," IEEE Transactions on Magnetics, Vol. 18, no. 2, pp. 319-324, March 1982.

[11] F. Marmin, S. Clenet, F. Piriou. "Error estimation of finite element solution in non linear magnetostatic 2D problem," IEEE Transactions on Magnetics, Vol. 34, no. 5, pp. 3268-3271, 1998.

[12] D.H. Mac, S. Clenet, J.C. Mipo, O. Moreau. "Solution for static field problem in random domains," IEEE Transactions on Magnetics, Vol. 46, no. 8, pp. 3385-3388, 2010.

[13] A. Bossavit. "Computational electromagnetism," Academic Press (Boston), 1998

\section{ACKNOWLEDGEMENTS}

This work is supported by the program MEDEE funded by the Nord Pas de Calais council and the European Community and supported in part by the National Science Foundation under Grant No. 1216927. 